\title{
Gene expression analysis after receptor tyrosine kinase activation reveals new potential melanoma proteins
}

\author{
Janka Teutschbein ${ }^{+1,4}$, Johannes M Haydn+11, Birgit Samans 2,5 , Michael Krause2 ${ }^{2}$ Martin Eilers ${ }^{3}$, Manfred Schartl1 and \\ Svenja Meierjohann*1
}

\begin{abstract}
Background: Melanoma is an aggressive tumor with increasing incidence. To develop accurate prognostic markers and targeted therapies, changes leading to malignant transformation of melanocytes need to be understood. In the Xiphophorus melanoma model system, a mutated version of the EGF receptor Xmrk (Xiphophorus melanoma receptor kinase) triggers melanomagenesis. Cellular events downstream of Xmrk, such as the activation of Akt, Ras, B-Raf or Stat5, were also shown to play a role in human melanomagenesis. This makes the elucidation of Xmrk downstream targets a useful method for identifying processes involved in melanoma formation.

Methods: Here, we analyzed Xmrk-induced gene expression using a microarray approach. Several highly expressed genes were confirmed by realtime PCR, and pathways responsible for their induction were revealed using small molecule inhibitors. The expression of these genes was also monitored in human melanoma cell lines, and the target gene FOSL 1 was knocked down by siRNA. Proliferation and migration of siRNA-treated melanoma cell lines were then investigated.

Results: Genes with the strongest upregulation after receptor activation were FOS-like antigen 1 (Fos/1), early growth response 1 (Egr1), osteopontin (Opn), insulin-like growth factor binding protein 3 (Igfbp3), dual-specificity phosphatase 4 (Dusp4), and tumor-associated antigen L6 (Taal6). Interestingly, most genes were blocked in presence of a SRC kinase inhibitor. Importantly, we found that FOSL1, OPN, IGFBP3, DUSP4, and TAAL6 also exhibited increased expression levels in human melanoma cell lines compared to human melanocytes. Knockdown of FOSL1 in human melanoma cell lines reduced their proliferation and migration.

Conclusion: Altogether, the data show that the receptor tyrosine kinase Xmrk is a useful tool in the identification of target genes that are commonly expressed in Xmrk-transgenic melanocytes and melanoma cell lines. The identified molecules constitute new possible molecular players in melanoma development. Specifically, a role of FOSL1 in melanomagenic processes is demonstrated. These data are the basis for future detailed analyses of the investigated target genes.
\end{abstract}

\section{Background}

Melanoma development is a complex process based on many epigenetic and genetic factors. The most frequent genetic changes in human melanoma are activating mutations in either BRAF or NRAS. This is often combined with inactivating mutations in phosphatase and tensin

\footnotetext{
*Correspondence: svenja.meierjohann@biozentrum.uni-wuerzburg.de 1 Department of Physiological Chemistry I, Biocenter, University of Wurzburg, Wurzburg, Germany

+ Contributed equally

Full list of author information is available at the end of the article
}

homologue $(P T E N)$ or cyclin-dependent kinase inhibitor 2 a $(C D K N 2 A)[1]$.

The search for other characteristics shared between human melanoma from different individuals has revealed the importance of several proteins influencing melanoma cell cycle progression, apoptosis, cell adhesion, and angiogenesis. Examples are cyclin-dependent kinase 4 (CDK4), AKT, $\beta$-catenin, melanoma inhibitory activity protein (MIA), and Ephrin-A1 (EFNA1) [1,2]. Still, the search for further melanoma-relevant genes is a promis- 
ing concept with potential therapeutic value, and several recent studies applying high-throughput gene expression profiling have associated previously unknown candidate genes with melanoma progression [3-5]. However, the comparability among different studies is low due to the variability of human tumor biopsies and the cultivationdependent changes in melanoma-derived cell lines.

By contrast, animal models represent genetic systems with well defined genetic background where tumorigenesis is initiated by known molecular events. In the Xiphophorus fish melanoma model, a single oncogenic epidermal growth factor receptor, termed Xiphophorus melanoma receptor kinase $(x m r k)$ is responsible for spontaneously developing melanoma [6].

Xmrk uses several signaling cascades that are also involved in human melanomagenesis, e.g. the phosphoinositide-3 kinase (PI3K) pathway and the RAS-RAFMAPK cascade. Other molecules, e.g. the signal transducer and activator of transcription 5 (STAT5) and osteopontin $(\mathrm{OPN})$, were first identified as essential mediators of Xmrk signaling and were subsequently shown to be relevant in human melanomagenesis as well [7-10]. These findings prompted us to search for novel Xmrk-regulated genes that may potentially play a role in human melanoma development.

It was shown many times that Xmrk signaling is highly comparable between its natural host cells - pigment cells from Xiphophorus - and mammalian cells that ectopically express the receptor [11-14]. Xmrk is permanently active due to its dimerization. However, to be able to differentiate between inactive and active receptor signaling, we are using the melanocytes cell line melan-a stably expressing a chimeric protein consisting of the extracellular part of EGFR ("HER") and the cytoplasmic part of Xmrk ("mrk") (melan-a "HERmrk" or shortly "Hm"). Melan-a cells lack endogenous EGFR, and the stimulation of $\mathrm{Hm}$ cells with EGF results in specific induction of Xmrk-dependent signaling pathways and tumorigenic transformation.

Here, we have analyzed gene expression profiles of stimulated versus unstimulated cells using a microarray approach. The genes with the strongest regulation in response to activated HERmrk were FOS-like antigen 1 (Fosl1), early growth response 1 (Egr1), osteopontin $(\mathrm{Opn})$, insulin-like growth factor binding protein 3 (Igfbp3), dual-specificity phosphatase 4 (Dusp4), and tumor-associated antigen L6 (Taal6). We investigated the pathways regulating these genes and analyzed their expression in human melanoma cell lines. We furthermore found that the knockdown of FOSL1 reduced proliferation and migration of human melanoma cell lines. Thus, this study reveals FOSL1 as new potential molecular player in melanomagenesis by using the Xmrk melanoma model.

\section{Methods}

\section{RNA isolation for microarray analysis}

Cells were starved for $72 \mathrm{~h}$ with DMEM containing 2.5\% dialyzed FCS. After stimulation with $100 \mathrm{ng} / \mathrm{ml}$ human EGF (hEGF) (tebu-bio, Le Perray en Yvelines, France) for indicated times, RNA was extracted from the cells using the RNeasy kit (Qiagen, Valencia, CA) according to the manufacturer's instructions. Only RNA samples with an $\mathrm{A}_{260} / \mathrm{A}_{280}$ ratio $>1.8$ were used for microarray hybridization.

\section{Microarray probe preparation and hybridization}

Transcriptional profiling was done on a microarray containing 21,168 DNA spots from the mouse cDNA library NIA $15 \mathrm{k}$ and 7.4 k Mouse cDNA Clone Set (National Institute on Aging, Bethesda, MD). Total RNA was purified with RNeasy spin columns (Qiagen). After mRNA amplification with MessageAmp II aRNA Kit (Ambion, Austin, TX USA), Cy3- and Cy5-labeled cDNA probes were generated using the CyScribe cDNA Post-Labelling Kit (GE Healthcare, Amersham Place, Little Chalfont England). The labeled probes were purified with QIAquick PCR Purification Kit (Qiagen, Hilden, Germany), combined in hybridization buffer $(0.2 \%$ SDS, $4.5 \times$ SSC) and hybridized on the microarray for $16 \mathrm{~h}$ at $55^{\circ} \mathrm{C}$. Finally, the chips were washed at a stringency of $0.1 \times$ SSC/0.1\% SDS, dried by centrifugation, scanned and quantified using Scan Array Express (Perkin Elmer, Waltham, MA USA).

\section{Data analysis}

Each experiment was performed as sandwich hybridization, i.e. instead of a coverslip, a second microarray slide was used. This provides a replicated measurement for each hybridization that can be used for quality control and that reduces technical variability. For each spot, median signal and background intensities for both channels were obtained. To account for spot differences, the background-corrected ratio of the two channels was calculated and $\log 2$ transformed. To balance the fluorescence intensities for $\mathrm{Cy} 3$ and $\mathrm{Cy} 5$ as well as to allow for comparison of expression levels across experiments, the raw data were standardized. We used the print-tip-LOWESS normalization to correct for inherent bias on each chip. Expression data and gene annotations were stored in Array Express http://www.ebi.ac.uk/arrayexpress/ (accession: E-MEXP-1311), which complies with MIAME (minimal information about a microarray experiment) guidelines. The $\mathrm{R}$ environment software http://www.rproject.org/ was used for data analysis. To find differently expressed genes, changes in mRNA expression levels in stimulated versus unstimulated cells were calculated for each gene. The normalized data were filtered due to strict 
quality criteria and analyzed using Microsoft Excel. For experimental comparisons, genes showing at least a twofold change were chosen.

\section{Cell culture}

Mouse melanocytes transfected with HERmrk [melan-a Hm cells, [13]] or with human EGF receptor (melan-a HER) were cultured as described previously [14]. The human immortalized melanocyte cell line Hermes 3a (kindly provided by D. Bennett) was grown in RPMI supplemented with penicillin $(400 \mathrm{U} / \mathrm{ml})$, streptomycin $(50$ $\mu \mathrm{g} / \mathrm{ml})$, L-glutamine $(300 \mu \mathrm{g} / \mathrm{ml})$, TPA $(200 \mathrm{nM})$, cholera toxin $(2 \mathrm{pM}), \mathrm{hSCF}(10 \mathrm{ng} / \mathrm{ml})$, endothelin $(10 \mathrm{nM})$, and $10 \%$ FCS, as previously described [15]. Human melanoma cell lines Mel Im, Mel Wei, Mel Juso, and SK-Mel-3 (kind gifts by A. Bosserhoff) as well as A375, A375M, DX-3, LT5.1, and SK-Mel-28 were maintained in DMEM supplemented with penicillin $(400 \mathrm{U} / \mathrm{ml})$, streptomycin $(50$ $\mu \mathrm{g} / \mathrm{ml}), \mathrm{L}$-glutamine $(300 \mu \mathrm{g} / \mathrm{ml})$ and $10 \%$ FCS. Normal human epidermal melanocytes (NHEM) derived from foreskin were obtained from PromoCell (Heidelberg, Germany) and grown in melanocyte growth medium MGM (PromoCell) under a humidified atmosphere of 5\% $\mathrm{CO}_{2}$ at $37^{\circ} \mathrm{C}$. NHEM cells were used between passages 3 and 6 .

\section{Expression analysis by realtime PCR and pathway analysis}

Cells were starved as described and subsequently stimulated with $100 \mathrm{ng} / \mathrm{ml} \mathrm{hEGF}$ for indicated times. RNA extraction from stimulated melan-a Hm cells and human cell lines was done using Total RNA Isolation Reagent (ABgene, Epsom, UK) as recommended by the manufacturer. For the identification of pathways regulating expression of candidate genes, the small molecule inhibitors AG1478 $(20 \mu \mathrm{M})$, PP2 $(20 \mu \mathrm{M})$, LY294002 $(10 \mu \mathrm{M})$, or U0126 $(10 \mu \mathrm{M})$, respectively, were applied one hour prior to hEGF stimulation. Cells without inhibitor treatment received the equivalent amount of DMSO. Primary data of these experiments are available on request. cDNA was prepared from total RNA using the RevertAid kit with random hexamer primers (Fermentas, Burlington, Canada). PCR primers were designed using Primer3 software version $0.4 .0 \mathrm{http} / / /$ frodo.wi.mit.edu/primer3/. PCR was carried out using the iCycler IQ (Bio-Rad, Hercules, $C A)$. Values for each gene were normalized to expression levels of $\beta$-actin (mouse cell lines) or ribosomal protein S14 (human cell lines). Primer sequences are available on request.

\section{Cell lysis and Western blotting}

Cells were lyzed and blotted as described [16]. Before probing with the reference antibody, the blots were stripped as described. Monoclonal anti-phosphotyrosine
(PY20) was from BD Biosciences (San José, CA). Phospho-p44/42 MAPK (Thr202/Tyr204) antibody was purchased from Cell Signaling Technology (Danvers, MA). Polyclonal antibodies against FOSL1 (N-17, C-12), EGR1 (C-19), OPN (P-18 and K-20), IGFBP3 (H-98), MKP2 (H67), and ERK2 (C-14) were from Santa Cruz Biotechnology (Santa Cruz, CA). The MYC-tag antibody (9B11) was from Cell Signaling Technology (Danvers, MA). Secondary antibodies were conjugated with horseradish peroxidase and were directed against mouse (Pierce, Rockford, IL), rabbit (Bio-Rad) or goat (Abcam, Cambridge, UK). TAAL6 mouse IgM antibody (10H6) was a kind gift from S. Roffler. Secondary antibody against mouse IgM was purchased from Rockland (Gilbertsville, PA).

\section{siRNA transfection}

One day before siRNA transfection, melanoma cells were seeded at a density of $3 \times 10^{4}$ cells per well of a 12-well plate. For human melanoma cells, commercially available siRNA against human FOSL1 as well as control siRNA (Smart Pool On Target Plus, Thermo Scientific) were used. siRNA was transfected using X-treme gene transfection reagent (Roche), according to the manufacturer's recommendations. Downregulation was monitored after $48 \mathrm{~h}$ by realtime PCR and Western blot analysis.

\section{BrdU incorporation analysis}

Melanoma cell lines A375 and Mel Juso were plated in triplicate $\left(2.5 \times 10^{3}\right.$ cells per well of a 96-well plate) in DMEM containing 10\% FCS. $48 \mathrm{~h}$ after siRNA treatment, cells were incubated with $10 \mu \mathrm{M}$ BrdU for $6 \mathrm{~h}$. BrdU incorporation was then quantified using a colorimetric BrdU cell proliferation ELISA, as recommended by the manufacturer (Roche).

\section{Transwell migration assay}

$2 \times 10^{4} \mathrm{~A} 375$ or Mel Juso cells were transfected with control- or FOSL1-specific siRNA. One day later, they were serum-starved in DMEM containing 1\% dialyzed FCS for $24 \mathrm{~h}$ and applied to the upper chamber of a transwell inlay (polycarbonate, $10 \mathrm{~mm}$ diameter, $8 \mu \mathrm{m}$ pores, Nunc). Migration was measured as described before [14]. The migration rate of the siFOSL1-treated cells was determined relative to the migration of control cells.

\section{Results}

Temporal gene expression profile after Xmrk activation

To obtain a detailed picture of the time course of Xmrkdependent gene expression, we compared unstimulated melan-a HERmrk cells to those stimulated for 15 minutes, $1 \mathrm{~h}, 2 \mathrm{~h}, 4 \mathrm{~h}, 8 \mathrm{~h}$ or $24 \mathrm{~h}$ with EGF.

Successful receptor activation was controlled by Western blot (Additional file 1, Figure S1a) and realtime PCR 
(Additional file 1, Figure S1b). Both assays revealed successful stimulation, visible by receptor- and MAPK phosphorylation as well as induction of the Xmrk target gene Opn.

Subsequent microarray analysis revealed the regulation of 1,273 genes at one or more time-points after HERmrk stimulation. A gene was considered to be regulated when changed twofold and more. The complete list of expression data and gene annotations is available at http:// www.ebi.ac.uk/arrayexpress/ (accession: E-MEXP-1311).

Regulated genes were categorized with respect to their molecular functions and biological processes according to the Gene Ontology (GO) terminology. Using the expression analysis systematic explorer (EASE) software [17], overrepresentation of gene ontology terms in the 1,273 regulated genes compared to the total number of genes assayed (21,168 spots on the chip) was calculated. Significantly enriched categories (EASE score $<0.05$ ) are listed in Additional file 2, Table S1. Among the biological processes, protein metabolism and protein modification were particularly enriched, indicating a high metabolic activity as expected from growth factor stimulated cells, and enhancement of signal transduction processes. The most overrepresented molecular function was nucleic acid binding, encompassing transcription factors and factors regulating nucleic acid stability.

For further analysis, we chose eleven genes which were assigned by UniGene and which displayed more than fourfold regulation at one or more time points. Cyr61, $I g f b p 3$, and Opn encode secreted proteins. SOS1 is a guanine nucleotide exchange factor. EGR1 and FOSL1 are transcription factors. EMP1 and TAAL6 are integral membrane proteins, whereas UBE2I and DUSP4 are cytosolic enzymes with ubiquitin-conjugating and phosphatase activity, respectively. Finally, the transcript with UniGene ID Mm.204306 has no assigned function. The time-dependent course of gene expression is depicted in a color map (Figure 1). Genes regulated at early time points were for example Cyr61 and Egr1, while Emp1 and Taal6 were regulated at late stimulation times.

To validate the microarray results for highly regulated genes, we used quantitative realtime PCR (Figure 2). The time course already observed in the microarray experiment was largely confirmed by realtime PCR analysis. Sos1, Ube2i, Cyr61 and Egr1 were mainly upregulated after short stimulation times and decreased later. The expression of Dusp4 was highest after $1 \mathrm{~h}$, but an upregulation in comparison to the unstimulated control was visible until $24 \mathrm{~h}$. In case of Igfbp3, the situation looked slightly different compared to the microarray experiment. While in the latter the gene was found to be upregulated after $15 \mathrm{~min}$ and again from 4 to $24 \mathrm{~h}$, the transcription induction was only visible at early times when analyzed by realtime PCR. For Fosl1, the expression was highest after $2 \mathrm{~h}$ (200-fold) and decreased later, similar to the situation observed in the microarray experiment. Expression levels of Emp1 were strongly increasing from 2 to $8 \mathrm{~h}$ and decreased to 4 -fold at $24 \mathrm{~h}$. The late-responding genes Opn, Taal6, and the unnamed gene product (UniGene ID Mm.204306, data not shown) steadily increased with the highest levels at $24 \mathrm{~h}$. However, as the gene with UniGene ID Mm.204306 was retired from UniGene during the course of our experiments, we omitted it from further analyses.

Altogether, in all cases except $I g f b p 3$, transcript induction as well as its time course was confirmed by realtime PCR.

\section{Identification of signaling pathways}

Previous analyses have revealed that Xmrk transfers signals via STAT5, the PI3K pathway, the RAS-RAF-MAPK cascade, and the cytoplasmic SRC kinase family member FYN [6]. To identify the pathways responsible for modulation of the chosen ten genes, we blocked known Xmrkinduced pathways using the small molecule inhibitors AG1478, U0126, PP2, or LY294002. Target gene expression in presence or absence of the inhibitors was analyzed by realtime PCR (Additional file 3, Table S2 and, for a schematic overview, Addditional file 4, Figure S2).

As expected, the inducing effects of EGF on all genes were abrogated when HERmrk was inhibited by AG1478. While regulation of Emp1, Fosl1, and Opn was both MEK- and SRC family kinase-dependent, induction of Sos1, Ube2I, Dusp4, and Taal6 was only restrained by inhibiting SRC-family kinases with PP2. Egr1 expression could only be decreased after MEK inhibition, and Cyr61 transcription was dependent on MEK and PI3K. Finally, expression of $I g f b p 3$ was decreased after application of each of the inhibitors.

\section{Expression of candidate genes in human melanoma cell lines}

For further analysis, we focused on six significantly regulated genes from four different functional groups: the transcription factors FOSL1 and EGR1, the secreted proteins $O P N$ and IGFBP3, the phosphatase DUSP4, and the membrane protein TAAL6. We monitored their expression levels in human melanoma cell lines compared to normal human epidermal melanocytes (NHEM). For this analysis we chose eight different cell lines, containing either activating N-RAS- (DX-3, LT5.1 and Mel Juso) or B-RAF mutations (A375, A375M, Mel Wei, Mel Im, SKMel-3 and SK-Mel-28).

Realtime PCR revealed a significantly higher expression of FOSL1, OPN, IGFBP3, DUSP4 and TAAL6 in most of the melanoma cell lines compared to normal melanocytes (Figure 3a). The sole exception was observed in case of 


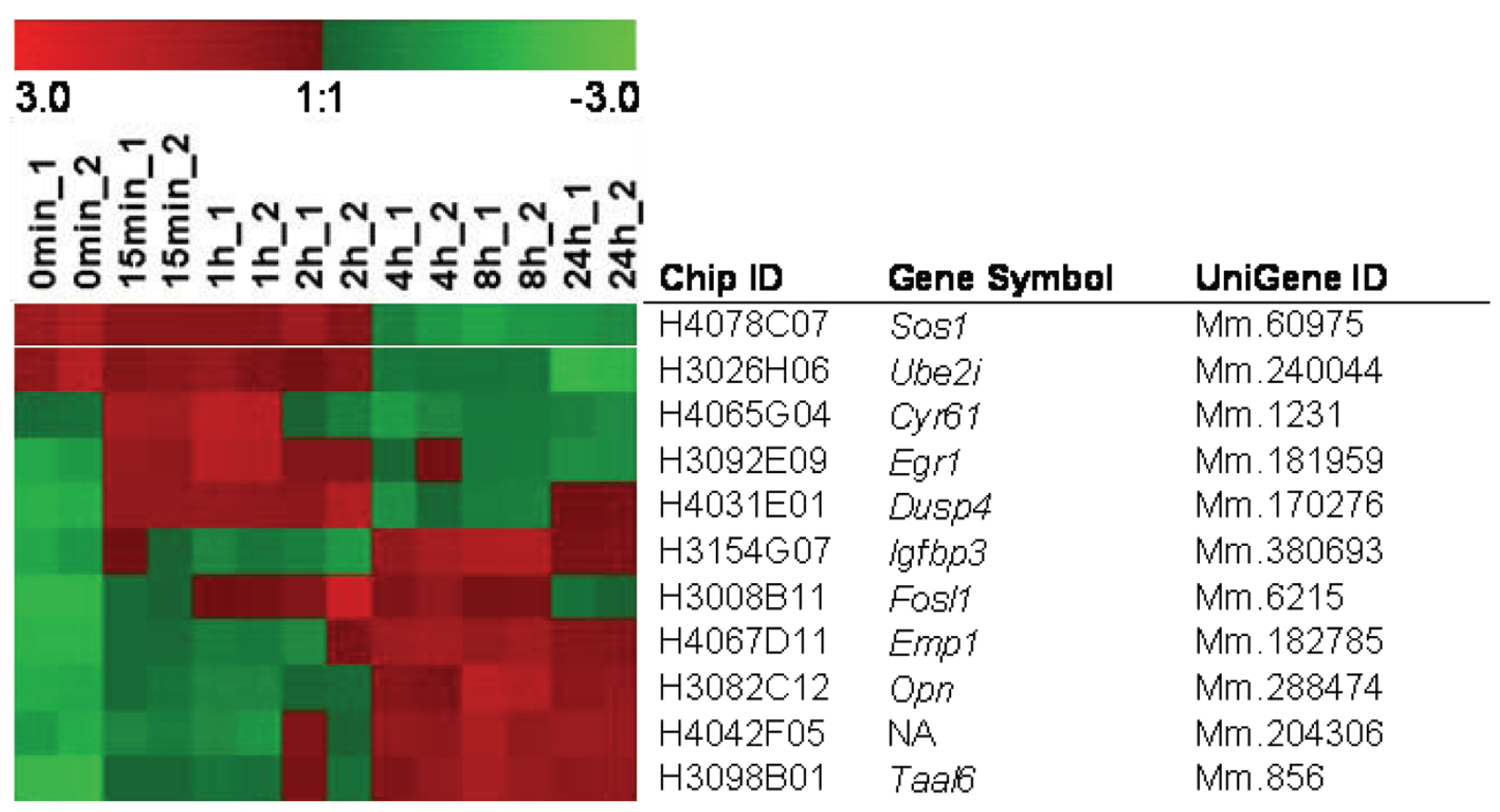

Figure 1 Color map of Xmrk-specific gene expression. Eleven highly regulated genes are itemized. RNA was extracted at time 0 (no hEGF) and at indicated time points after hEGF addition. The color code shows differences in the expression of the eleven genes regulated $>4$-fold in the range of +3 (red) to -3 (green).

Sos1, son of sevenless homolog 1; Ube2i, ubiquitin-conjugating enzyme E21; Cyr61, cysteine rich angiogenic inducer 61; Egr1, early growth response 1; Dusp4, dual specificity phosphatase 4; Igfbp3, insulin-like growth factor binding protein 3; Fos/1, FOS-like antigen 1; Emp 1, epithelial membrane protein 1; Opn, osteopontin; Taal6, tumor-associated antigen L6. NA: not annotated.

EGR1, where only A375M cells displayed a significant upregulation. At the level of protein, NHEM displayed almost no expression of most of the proteins, whereas in the majority of examined melanoma cell lines FOSL1, IGFBP3 and DUSP4 were strongly expressed (Figure 3b). EGR1, OPN and TAAL6 were expressed in at least two third of the cell lines, but not in NHEM cells.

As Xmrk is an orthologue of the human EGF receptor, we wanted to elucidate if the upregulation of the identified target genes is a shared property between human EGFR and Xmrk/HERmrk. Of the 10 C-terminal SH2 docking sites of human EGFR, most of them with overlapping substrate specifity, 7 are conserved in Xmrk, suggesting at least partial functional similarity. The melanoma cell line A375 reportedly expresses human EGFR and responds to addition of EGF [18]. When induction of FOSL1, EGR1, OPN, IGFBP3, DUSP4 and TAAL6 was monitored after EGF stimulation between 15 minutes and $24 \mathrm{~h}$, only the fast responding FOSL1 and EGR1 genes were found to be induced (Figure 4a and data not shown). Compared to HERmrk-expressing melanocytes, FOSL1 upregulation was weaker in A375, while EGR1 induction was even stronger (compare Figure 4a and Figure 2). As A375 cells express oncogenic BRAFV600E and already underwent the process of transformation, it is possible that ongoing endogenous aberrant signaling concealed EGFR stimulation in this cell line. For this reason, and to gain a better comparison to the untransformed melan-a HERmrk cells, we used melan-a cells stably transfected with human EGFR ("melan-a HER") and performed an experiment similar to the one performed with A375 cells (Figure 4b). Here, all investigated genes except $I g f b p 3$ were upregulated in response to EGF. Apart from the downregulated Opn and Taal6 values at $24 \mathrm{~h}$, the extent and time course of stimulation were comparable between HERmrk and HER stimulation (Figures 2 and $4 b$ ).

Among the genes identified, the protein encoded by FOSL1 constitutes an interesting candidate with a potential effect on melanoma biology. It is part of the AP-1 complex, which is a functional downstream target of the MAP kinase pathway that is commonly activated in melanoma [19]. Furthermore, c-JUN, which might be a potential binding partner for FOSL1 in the AP-1 complex, is highly expressed in most melanoma and is required for tumor transformation [20-22]. The human protein atlas database constitutes a platform which offers an extensive amount of protein expression data gained from a large 

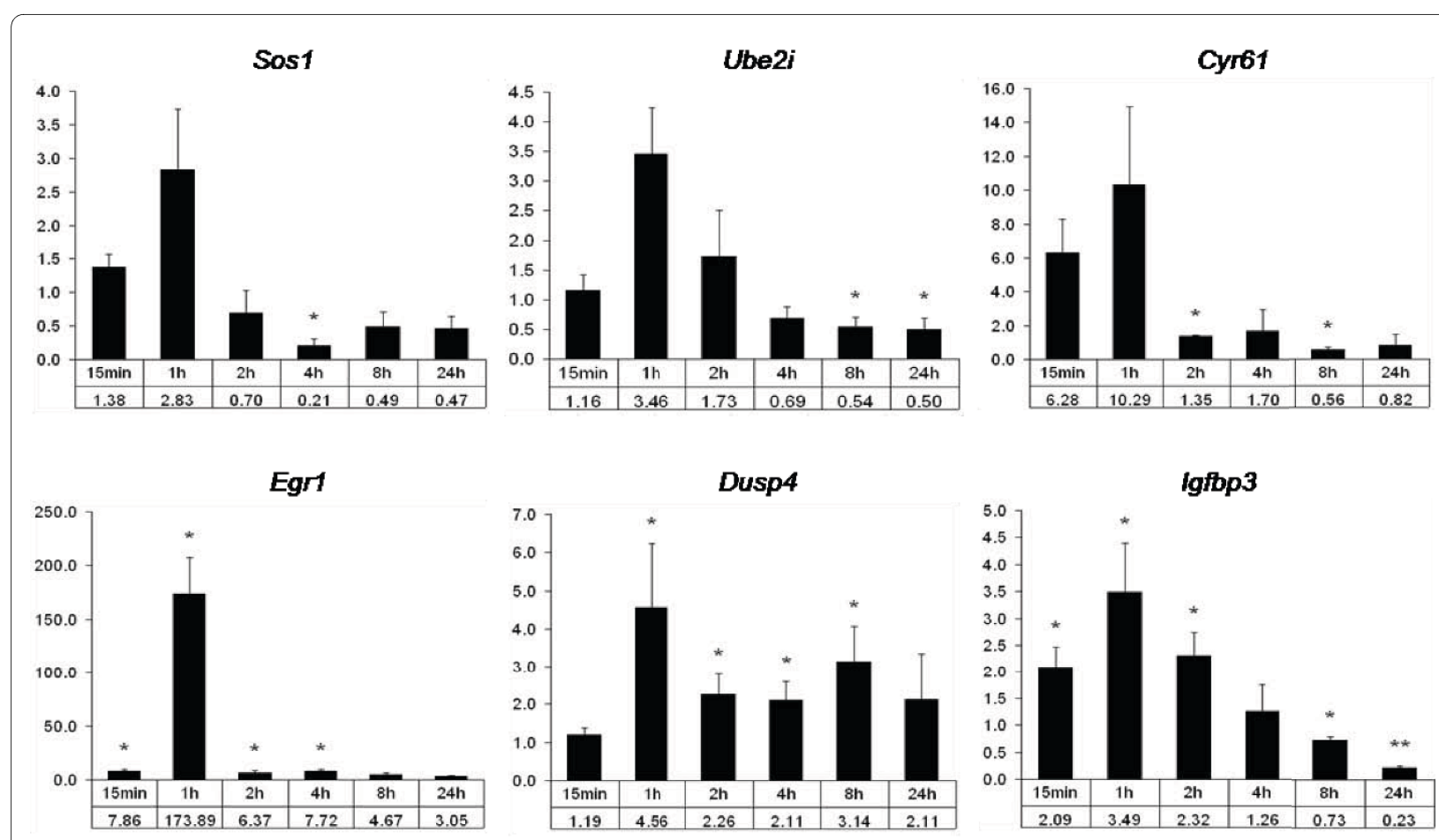

Fosl1

Emp1
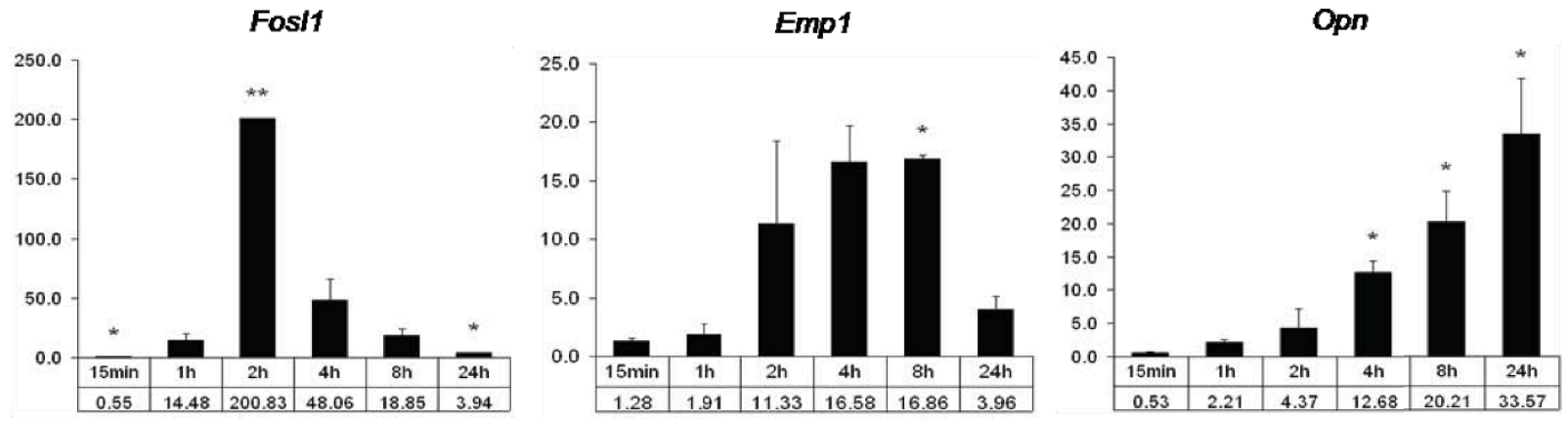

Taal6

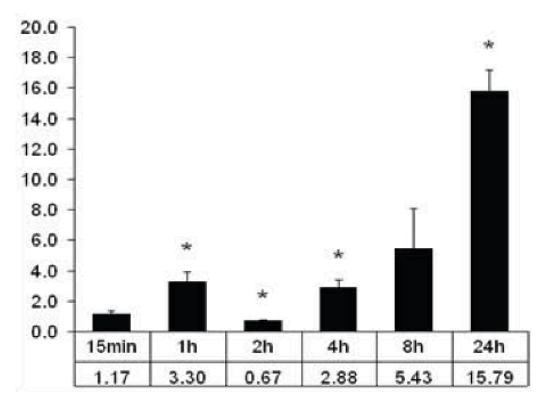

Figure 2 Validation of microarray results of ten candidate genes by quantitative realtime PCR. RNA was extracted at indicated time points after HERmrk stimulation with hEGF. Expression of candidate genes was compared to unstimulated cells. The fold change of transcript, referred to the unstimulated control, which is set as 1 , is indicated on the y axis. Mouse $\beta$-actin served as reference gene. Significant regulation $(p<0.05)$ is marked by an asterisk; two asterisks indicate highly significant regulation $(p<0.001)$ (Student's t-test, paired, one-tailed). 


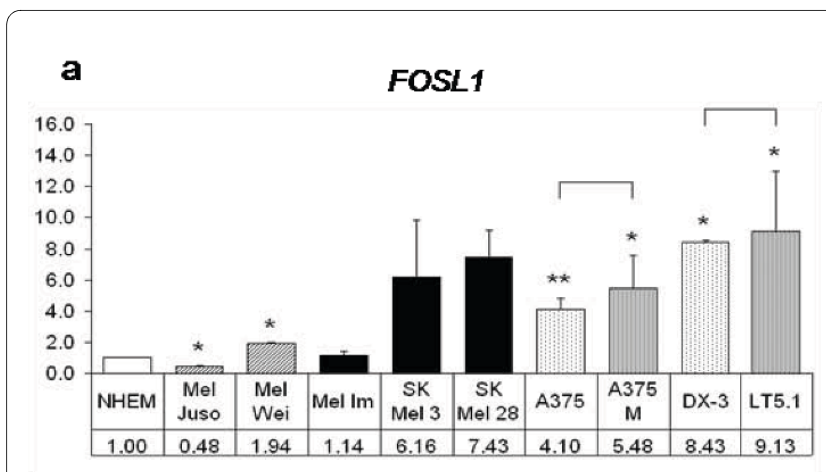

OPN

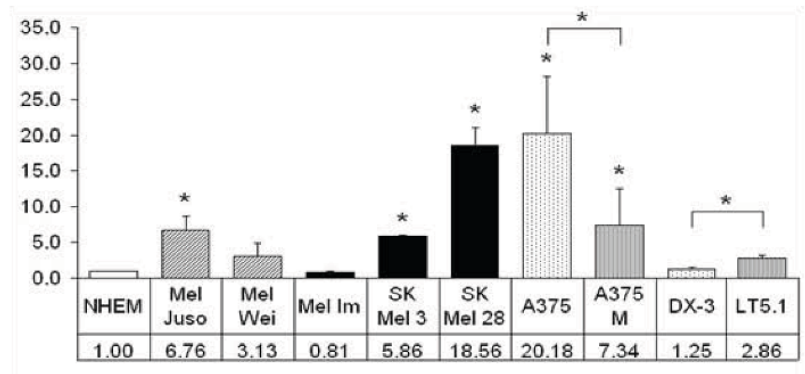

DUSP4

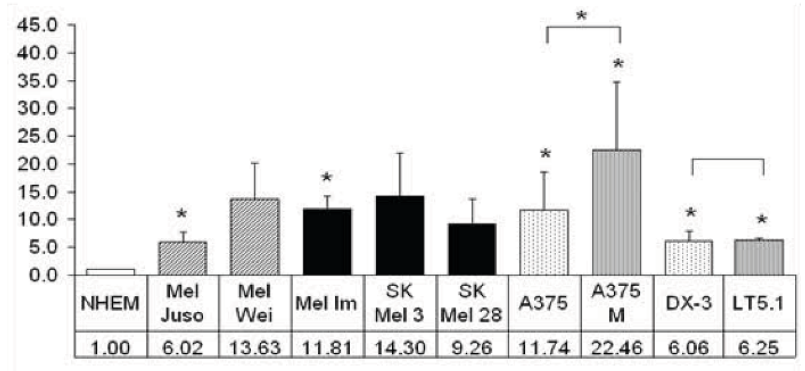

b

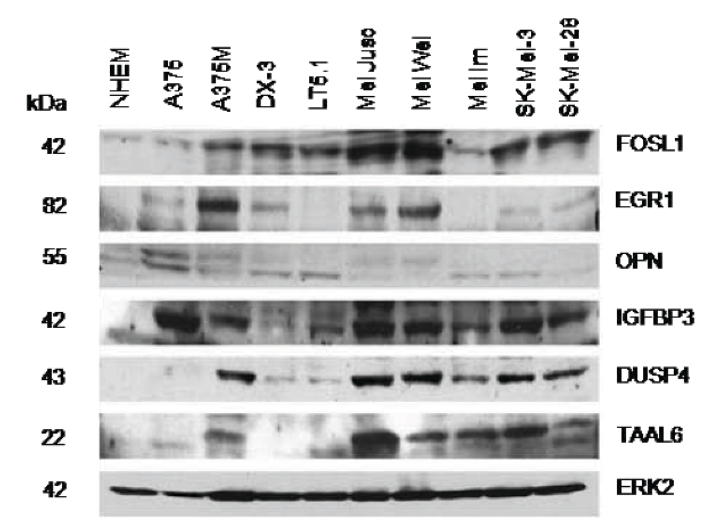

Figure 3 Expression of candidate genes in human melanoma cell lines. $a$, Realtime PCR was performed to determine FOSL1, EGR1, OPN, IGFBP3, DUSP4 and TAAL6 expression in human melanoma cell lines compared to NHEM cells. Fold change of transcript, referred to NHEM cells, is indicated. $\beta$-actin was used as reference gene. Significant $(p<0.05)$ or highly significant $(p<0.001)$ regulation are marked by one or two asterisks, respectively (Student's t-test, paired, one-tailed).

b. Western blot analysis of the candidate genes in human melanoma cell lines. Expression of ERK2 served as loading control. Please note that the order of cell lines differs from A. On the OPN blot, the lower band represents the OPN protein. For TAAL6, the upper band corresponds to the protein of the expected size. 
a

FOSL1

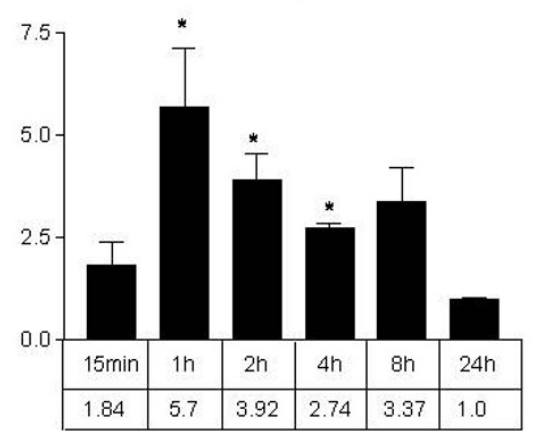

Fosi1

b

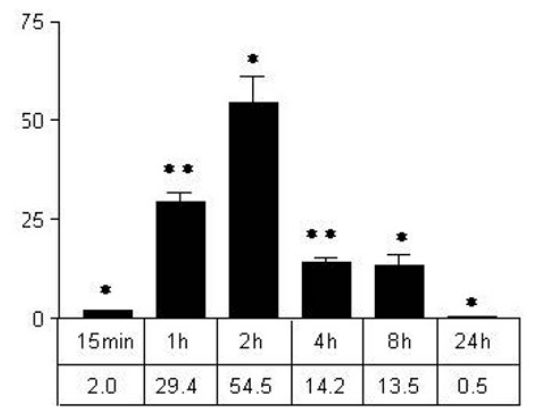

EGR1

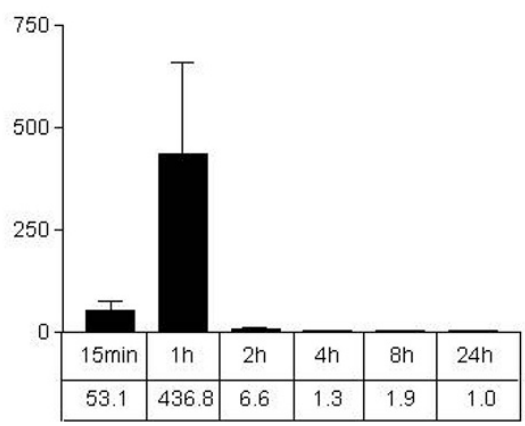

Opn

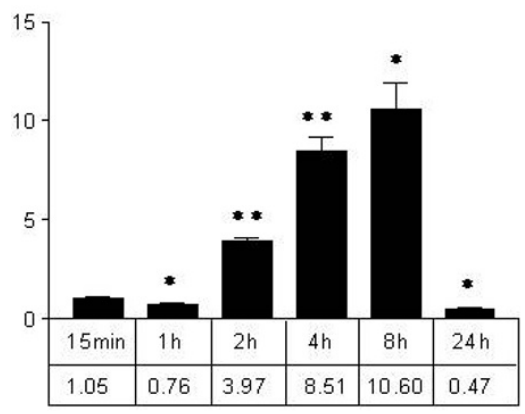

Dusp4

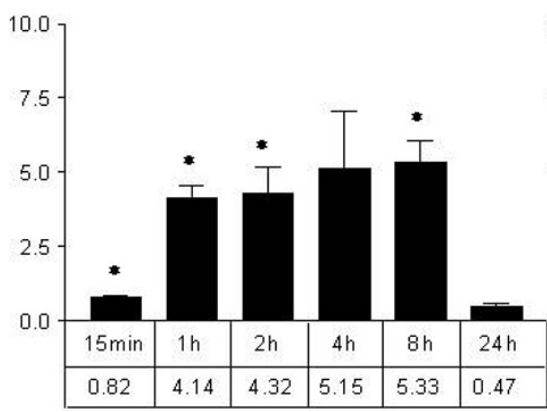

Egr

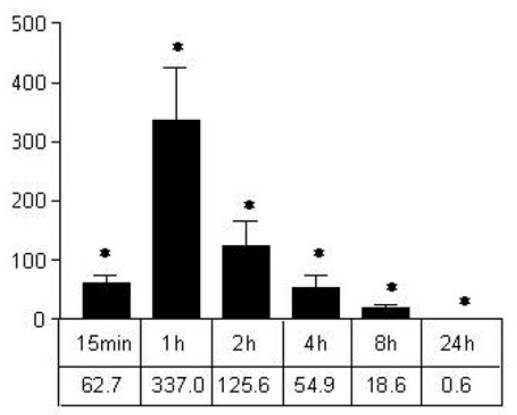

Figure 4 Expression of candidate genes in response to stimulation of human EGFR. Realtime PCR was performed to determine expression levels of FOSL1 and EGR1 in the human melanoma cell line A375 (a) or expression levels of Fos/1, Egr1, Opn, Dusp4, and Taal6 in HER-transgenic melan-a cells (b), stimulated with EGF for the indicated times. The fold change of transcript, referred to the unstimulated controls, is indicated on the $y$ axis. Murine and human $\beta$-actin were used as reference genes. Significant $(p<0.05)$ or highly significant $(p<0.001)$ regulation are marked by one or two asterisks, respectively (Student's t-test, paired, two-tailed).

variety of normal human tissues, cancer tissues and cell lines $[23,24]$. Here, FOSL1 expression is low or nondetectable in most tissues, and moderate in epidermal skin cells. Among melanoma tissues, two thirds express moderate or high levels of the protein, and both melanoma cell lines investigated also show high expression http://www.proteinatlas.org/ tissue profile.php?antibody id $=4396 \& \mathrm{~g}$ no=ENSG0000 0175592. These data confirm our own observations, namely the increase of FOSL1 expression in transformed or activated pigment cells. In our study, FOSL1 protein levels were not only upregulated in mouse melanocytes expressing HERmrk, but were also elevated in human melanoma cell lines compared to the human melanocyte 
a

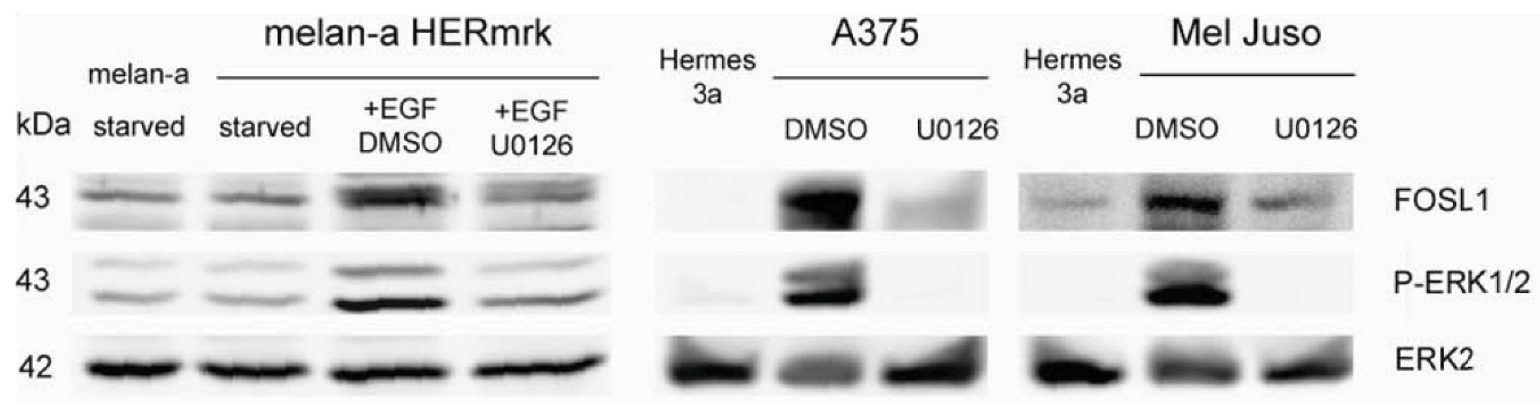

b
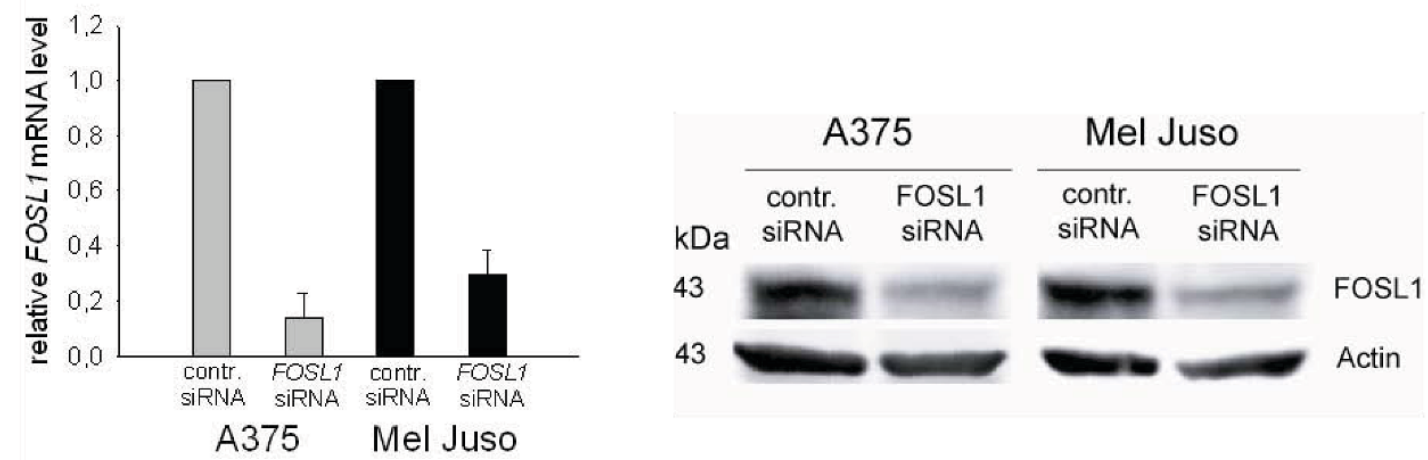

C

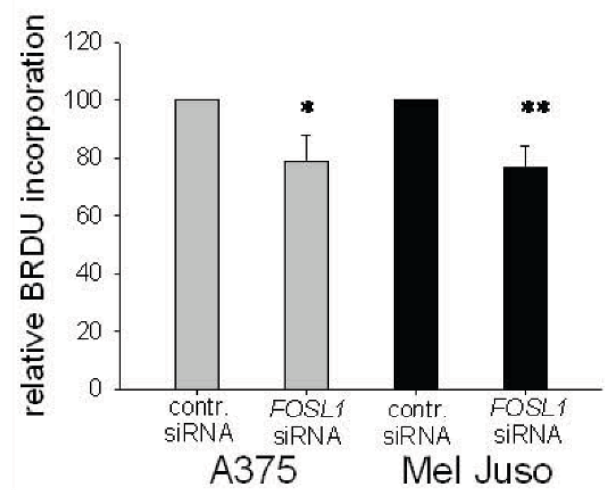

d

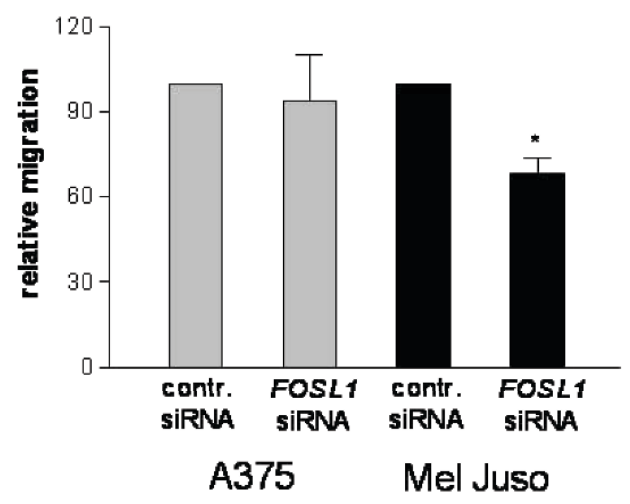

Figure 5 MAPK-pathway dependent expression of FOSL1 and effects of FOSL1 knockdown on proliferation and migration of melanoma cells A375 and Mel Juso. a, Western blot analysis of FOSL1 levels in murine melanocytes (left) and human melanocytes and melanoma cell lines (right). Murine melan-a and melan-a HERmrk cells were starved for 2 days before EGF and U0126 were added for 24 hours. Human melanoma cells were treated with U0126 for 24 hours. P-ERK1/2 levels were used to verify MEK inhibition, ERK2 was used as reference.

$b$, Realtime PCR analysis (left) and Western blot analysis (right) of FOSL1 expression levels after treatment of the indicated cell lines with control siRNA or FOSL1-specific siRNA. For realtime PCR analysis, control siRNA-treated FOSL1 levels were set as 1. For the Western blot, $\beta$-actin was used as reference. c, BrdU incorporation of A375 and Mel Juso melanoma cells transfected with control siRNA or FOSL1-specific siRNA.

d, Migration of A375 and Mel Juso cells treated with FOSL1-specific siRNA relative to control siRNA-treated cells.

Significant $(p<0.05)$ or highly significant $(p<0.001)$ differences between control and FOSL1 siRNA treated cells are marked by one or two asterisks, respectively (Student's t-test, paired, two-tailed). 
cell line Hermes3a (Figure 5a) and NHEM cells (Figure $3 \mathrm{~b})$. Inhibition of MEK strongly reduced FOSL1 protein in HERmrk-transgenic cells as well as in the human cell lines A375 and Mel Juso (Figure 5a). This suggests that MAPK pathway activation by BRAF ${ }^{\mathrm{V} 600 \mathrm{E}}$ (as in A375) and by NRASQ61K (as in Mel Juso) is important in maintaining FOSL1 expression. To investigate the effect of FOSL1 on melanoma growth, we downregulated FOSL1 in the melanoma cell lines A375 and Mel Juso using siRNA (Figure $5 b)$. Proliferation was monitored by BrdU incorporation assay, which indicates the number of cells in $\mathrm{S}$ phase (Figure 5c). In both cell lines, BrdU incorporation was significantly reduced in presence of FOSL1 siRNA (79 and 77\%, respectively). Furthermore, the migration capacity of $\mathrm{Mel}$ Juso cells was reduced to $66 \%$ (Figure $5 \mathrm{~d}$ ).

\section{Discussion}

Here we describe the regulation of potential novel melanoma candidate genes using an inducible version of the EGFR orthologue Xmrk.

Ten of the most strongly regulated genes were examined in presence of pathway inhibitors to determine the respective signal transduction chain responsible for their regulation. Eight out of ten genes were at least partly controlled by members of the SRC kinase family, while six genes were induced by the MAPK pathway and only two genes by the PI3 kinase pathway. Usually, gene regulation was reduced by inhibition of more than one pathway, which indicates an interplay between the simultaneously activated pathways. Although we could only perform the inhibitor analysis with a small group of genes, which is not representative of the overall mode of gene regulation, it was surprising that such a high gene number was regulated by SRC kinases. From previous studies it is known that FYN is the only SRC family kinase member that is directly activated by Xmrk [25]. FYN prevents inactivation of MAPK by reducing MAPK phosphatase 1 (MKP1) expression, but also stabilizes the complex between Xmrk and the p85 subunit of PI3K [13,26]. This might explain why in our study many genes are not only affected by SRC family kinase inhibition, but also by blocking MEK or PI3K. Although the effect of SRC kinases on gene expression has not yet been investigated in human melanoma, accumulating data indicate that this pathway plays a vital role for the malignant cells. Specifically, it was shown by kinase activity profiling that SRC is activated in primary human melanoma and its inhibition leads to reduced growth [27]. Activated SRC was also detected in cutaneous, mucosal and metastatic melanoma, and its inhibition by dasatinib or bosutinib blocked the growth of several melanoma cell lines $[28,29]$. Furthermore, an active SRC family kinase-focal adhesion kinase complex, responsible for migration and metastatic spread, was described both for the Xmrk model and for human mela- noma $[14,30]$. Together with our own data, this depicts an important melanomagenic role for SRC kinases in general and raises the necessity to further scrutinize SRC family kinase-dependent gene regulation in human melanoma.

Among the identified genes, expression of Egr1, Dusp4, Igfbp3, Fosl1, Opn, and Taal6 were investigated more closely. Importantly, five of these genes have been found to be upregulated in several different melanoma microarray studies. Recently, B-RAFV600E-dependent genes were analysed by MEK inhibition or BRAF siRNA in human melanoma cell lines, revealing regulation of the transcription factors FOSL1 and EGR1 by this pathway [31]. Interestingly, EGR1 belongs to a distinct group of salivary marker genes expressed in melanoma-bearing mice [32]. IGFBP3 was more strongly expressed in metastatic and cutaneous melanoma compared to melanocytes [33]. Its function seems to depend on the cellular context, as both anti- and pro-tumorigenic roles were attributed to this protein $[34,35]$.

DUSP4 and OPN were upregulated in cutaneous melanoma in comparison to normal skin or benign nevi [5]. DUSP4 is a dual phosphatase capable of dephosphorylating p38, JNK1 and ERK1/2, though JNK1 seems to be the preferred target in vitro [36]. Its upregulation may simply reflect counterregulation of the ERK1/2 pathway in melanoma, but has not yet been investigated more closely. $\mathrm{OPN}$, on the other hand, is a well-known melanoma marker $[37,38]$ and served as internal control in our studies.

Finally, in a murine melanoma model with xenografted A375 cells, EGR1 and OPN expression was enhanced in cells derived from metastatic melanoma compared to the parental cell line as well as in metastatic melanoma versus primary melanoma [39]. The original data of all abovementioned microarray studies are available at http:// www.oncomine.org.

Due to the important role of the AP-1 complex in human melanoma, the lack of information on FOSL1 function in this tumor type, and the MAPK pathway dependent induction of FOSL1 in melanoma cell lines, we investigated the effect of FOSL1 knockdown on the proliferation of two melanoma cell lines and found a significant inhibition of cells entering the $S$ phase, which was not due to the induction of apoptosis (data not shown). Also, migration of Mel Juso cells was decreased after FOSL1 knockdown. Its function in melanoma was not described previously, but pro-tumorigenic roles of FOSL1 were reported for other solid cancers $[40,41]$. Oncogenic EGFRvIII in glioblastoma cells induces FOSL1 [41], and it modulates the malignant features of glioma cells, so it was suggested as target for therapeutic interventions against malignant gliomas [42]. An oral DNA vaccine directed against FOSL1 has been demonstrated to effec- 
tively suppress tumor growth, angiogenesis and metastasis in mice injected with breast carcinoma cells [43].

In summary, we used the high overlap between pathways downstream of Xmrk and established human melanoma pathways for the search of new melanoma-relevant target genes. Our gene and protein expression results indicate that Xmrk serves as a suitable model oncogene for this purpose. As a proof of principle, we investigated the AP-1 complex component FOSL1 in more detail. We found that the gene is similarly regulated in a MAPKdependent manner by Xmrk and by human melanoma oncogenes. Importantly, we also could demonstrate a pro-tumorigenic role of FOSL1 in human melanoma cell lines, thus confirming the Xmrk oncogene as instrumental in the search of new melanoma players.

\section{Conclusions}

Here we show the receptor tyrosine kinase Xmrk is a valuable tool to identify target genes and proteins that are commonly shared between Xmrk-transgenic melanocytes and human melanoma cell lines. Interestingly, of the ten most strongly upregulated genes, the majority was regulated by SRC kinases, followed by the MAPK pathway. The transcription factor component gene FOSL1 also belongs to this group of genes. By knocking down FOSL1, we could demonstrate a pro-proliferative and pro-migratory function of this protein in melanoma cell lines. The presented data reveal new potential melanoma-relevant genes that can now be investigated for their melanomagenic function in detail.

\section{List of abbreviations used}

AKT: v-akt murine thymoma viral oncogene homolog; AP-1: activator protein 1; BRAF: v-raf murine sarcoma viral oncogene homolog B1; CDKN2A: cyclin dependent kinase inhibitor 2A; CYR61: cysteine-rich, angiogenic inducer 61; DUSP4: dual specificity phosphatase 4; EGF: epidermal growth factor; EGFR: epidermal growth factor receptor; EGR1: early growth response 1; EMP1: epithelial membrane protein 1; ERK: extracellular regulated MAP kinase; FOSL1: FOS-like antigen 1; FYN: FYN oncogene related to SRC, FGR, YES; HER: human epidermal growth factor receptor; HERmrk: chimera of HER and Xmrk; hSCF: human stem cell factor; IGFBP3: insulin-like growth factor binding protein 3; JNK1: JUN Nterminal Kinase 1; c-JUN: c-JUN oncogene; MAPK: mitogen-activated protein kinase; MKP-1: MAK kinase phosphatase 1; NHEM: normal human epidermal melanocyres; NRAS: neuroblastoma RAS viral (v-ras) oncogene homolog; OPN: osteopontin; PI3K: phosphoinositide-3-kinase; PTEN: phosphatase and tensin homolog; siRNA: small inhibitory RNA; SOS1: son of sevenless homolog 1; SRC: v-src sarcoma (Schmidt-Ruppin A-2) viral oncogene homolog; STAT5: signal transducer and activator of transcription 5; TAAL6: tumor-associated antigen L6; TPA: 12-O-Tetradecanoylphorbol-13acetate; UBE2I: ubiquitin-conjugating enzyme E2I; Xmrk: Xiphophorus melanoma receptor kinase

\section{Additional material}

\begin{abstract}
Additional File 1 Figure S1 Activation of the chimeric receptor HERmrk in melan-a cells. $a$, Stimulation of HERmrk with hEGF for indicated time periods resulted in autophosphorylation of the receptor (top) and phosphorylation of the downstream factor MAPK (bottom). b. Gene expression of the known Xmrk target Opn was induced after activation of HERmrk with hEGF. The fold change of transcript, referred to the unstimulated control, which is set as 1 , is indicated on the $y$ axis. Murine $\beta$-actin served as reference gene.

Additional file 2 Table S1 Expression analysis systematic explorer (EASE) report of differentially expressed genes. EASE analysis was performed with genes that were regulated $>2$-fold in microarray analysis. Only selected categories with an EASE score $<0.05$, i.e. categories with enrichment of differentially expressed genes, are listed. The number of regulated genes belonging to the category is quoted in the column List Hits.

Additional file 3 Table S2 Pathways for regulation of genes by activated HERmrk. Time and manner (up or down) of maximal regulation are itemized in the third column. Small molecule inhibitors AG1478, U0126, PP2, or LY294002 were applied to inhibit HERmrk, the MAPK kinase MEK, SRC-family kinases, or PI3K, respectively. Effects of inhibitors on expression of the candidate genes at indicated time points were monitored by realtime PCR. "+" indicates inhibition of Xmrk-dependent gene expression changes, "-" symbolizes that there was no effect.

Additional file 4 Figure S2 Schematic overview of the pathways induced by HERmrk and the subsequent induction of indicated genes, as shown in this manuscript.. Genes marked with an asterisk were only induced by one of the investigated pathways, while the induction of genes without asterisk was effected by three (Igfbp3) or two pathways (all other genes). The inhibitors used in this manuscript are depicted in red. AG1478 inhibits EGFR and its orthologues, including Xmrk. U0126 blocks MEK, LY294002 inhibits PI3 kinase, and PP2 inhibits SRC family kinases (FYN being the only one activated by Xmrk).
\end{abstract}

\section{Competing interests}

The authors declare that they have no competing interests.

\section{Authors' contributions}

SM and MS conceived and designed the experiments. JMH and JT performed all cell culture based analyses. BS and MK performed microarray analysis and data analysis. SM and JT wrote the manuscript. JMH, ME, and MS helped writing the manuscript and contributed to discussions. All authors read and approved the final manuscript.

\section{Acknowledgements}

We thank Anita Hufnagel for excellent technical assistance. Futhermore, we gratefully acknowledge the following people: Jochen Wilhelm kindly provided us the R package for microarray data analysis. The TAAL6 antibody (10H6) was donated by Steve Roffler. Melanoma cell lines and useful information on the cell lines were provided by courtesy of Anja Bosserhoff.

This work was supported by Transregio 17 ("Ras-dependent pathways in human cancer"), and Deutscher Melanomverbund (Deutsche Krebshilfe).

\section{Author Details}

1Department of Physiological Chemistry I, Biocenter, University of Wurzburg, Wurzburg, Germany, Institute of Molecular Biology and Tumor Research (IMT), University of Marburg, Marburg, Germany, ${ }^{3}$ Department of Physiological Chemistry II, Biocenter, University of Wurzburg, Wurzburg, Germany, 4Department of Molecular and Applied Microbiology, Leibniz-Institute for Natural Product Research and Infection Biology - Hans-Knoell-Institute, Jena, Germany and 5Biometry and Population Genetics, Justus Liebig University Giessen, Germany 
Received: 13 April 2010 Accepted: 21 July 2010

Published: 21 July 2010

\section{References}

1. Chin $\mathrm{L}:$ The genetics of malignant melanoma: lessons from mouse and man. Nat Rev Cancer 2003, 3(8):559-570.

2. Bosserhoff AK: Novel biomarkers in malignant melanoma. Clin Chim Acta 2006, 367(1-2):28-35.

3. Bittner $M$, Meltzer $P$, Chen $Y$, Jiang $Y$, Seftor E, Hendrix M, Radmacher M, Simon R, Yakhini Z, Ben-Dor A, Sampas N, Dougherty E, Wang E, Marincola F, Gooden C, Lueders J, Glatfelter A, Pollock P, Carpten J, Gillanders E, Leja D, Dietrich K, Beaudry C, Berens M, Alberts D, Sondak V: Molecular classification of cutaneous malignant melanoma by gene expression profiling. Nature 2000, 406(6795):536-540.

4. Hoek K, Rimm DL, Williams KR, Zhao H, Ariyan S, Lin A, Kluger HM, Berger AJ, Cheng E, Trombetta ES, WU T, Niinobe M, Yoshikawa K, Hannigan GE, Halaban R: Expression profiling reveals novel pathways in the transformation of melanocytes to melanomas. Cancer Res 2004 64(15):5270-5282.

5. Talantov D, Mazumder A, Yu JX, Briggs T, Jiang Y, Backus J, Atkins D, Wang $Y$ : Novel genes associated with malignant melanoma but not benign melanocytic lesions. Clin Cancer Res 2005, 11(20):7234-7242.

6. Meierjohann S, Schartl M: From Mendelian to molecular genetics: the Xiphophorus melanoma model. Trends Genet 2006, 22:654-661.

7. Geissinger E, Weisser C, Fischer P, Schartl M, Wellbrock C: Autocrine stimulation by osteopontin contributes to antiapoptotic signalling of melanocytes in dermal collagen. Cancer Res 2002, 62(16):4820-4828

8. Morcinek JC, Weisser C, Geissinger E, Schartl M, Wellbrock C: Activation of STAT5 triggers proliferation and contributes to anti-apoptotic signalling mediated by the oncogenic Xmrk kinase. Oncogene 2002, 21(11):1668-1678.

9. Wellbrock C, Weisser C, Hassel JC, Fischer P, Becker J, Vetter CS, Behrmann I, Kortylewski M, Heinrich PC, Schartl M: STAT5 contributes to interferon resistance of melanoma cells. Curr Bio/ 2005, 15(18):1629-1639.

10. Zhou Y, Dai DL, Martinka M, Su M, Zhang Y, Campos El, Dorocicz I, Tang L, Huntsman D, Nelson C, Ho V, Li G: Osteopontin expression correlates with melanoma invasion. J Invest Dermato/ 2005, 124(5):1044-1052.

11. Wellbrock C, Fischer P, Schartl M: Receptor tyrosine kinase Xmrk mediates proliferation in Xiphophorus melanoma cells. Int $\mathrm{J}$ Cancer 1998, 76(3):437-442.

12. Wellbrock C, Schartl M: Multiple binding sites in the growth factor receptor Xmrk mediate binding to p59fyn, GRB2 and Shc. Eur J Biochem 1999, 260(1):275-283.

13. Wellbrock C, Weisser C, Geissinger E, Troppmair J, Schartl M: Activation of p59(Fyn) leads to melanocyte dedifferentiation by influencing MKP-1regulated mitogen-activated protein kinase signaling. J Biol Chem 2002, 277(8):6443-6454.

14. Meierjohann S, Wende E, Kraiss A, Wellbrock C, Schartl M: The oncogenic epidermal growth factor receptor variant xiphophorus melanoma receptor kinase induces motility in melanocytes by modulation of focal adhesions. Cancer Res 2006, 66(6):3145-3152.

15. Gray-Schopfer VC, Cheong SC, Chong H, Chow J, Moss T, Abdel-Malek ZA, Marais R, Wynford-Thomas D, Bennett DC: Cellular senescence in naevi and immortalisation in melanoma: a role for p16? Br J Cancer 2006, 95(4):496-505.

16. Leikam C, Hufnagel A, Schartl M, Meierjohann S: Oncogene activation in melanocytes links reactive oxygen to multinucleated phenotype and senescence. Oncogene 2008, 27(56):7070-7082.

17. Hosack DA, Dennis G Jr, Sherman BT, Lane HC, Lempicki RA: Identifying biological themes within lists of genes with EASE. Genome Biol 2003, 4(10):R70.

18. Mirmohammadsadegh A, Hassan M, Bardenheuer W, Marini A, Gustrau A, Nambiar S, Tannapfel A, Bojar H, Ruzicka T, Hengge UR: STAT5 phosphorylation in malignant melanoma is important for survival and is mediated through SRC and JAK1 kinases. J Invest Dermatol 2006, 126(10):2272-2280.

19. Poser I, Bosserhoff AK: Transcription factors involved in development and progression of malignant melanoma. Histol Histopathol 2004, 19(1):173-188.
20. Lopez-Bergami P, Huang C, Goydos JS, Yip D, Bar-Eli M, Herlyn M, Smalley KS, Mahale A, Eroshkin A, Aaronson S, Ronai Z: Rewired ERK-JNK signaling pathways in melanoma. Cancer Cell 2007, 11(5):447-460.

21. Lopez-Bergami P, Kim H, Dewing A, Goydos J, Aaronson S, Ronai ZA: CJUN regulates PDK1 transcription: implication for AKT and PKC activities and melanoma tumorigenesis. J Biol Chem 2009, 285(2):903-913.

22. Gurzov EN, Bakiri L, Alfaro JM, Wagner EF, Izquierdo M: Targeting c-Jun and JunB proteins as potential anticancer cell therapy. Oncogene 2008, 27(5):641-652.

23. Berglund L, Bjorling E, Oksvold P, Fagerberg L, Asplund A, Szigyarto CA, Persson A, Ottosson J, Wernerus H, Nilsson P, Lundberg E, Sivertsson A, Navani S, Wester K, Kampf C, Hober S, Ponten F, Uhlen M: A genecentric Human Protein Atlas for expression profiles based on antibodies. Mol Cell Proteomics 2008, 7(10):2019-2027.

24. Uhlen M, Bjorling E, Agaton C, Szigyarto CA, Amini B, Andersen E, Andersson AC, Angelidou P, Asplund A, Asplund C, Berglund L, Bergström K, Brumer H, Cerjan D, Ekström M, Elobeid A, Eriksson C, Fagerberg L, Falk R, Fall J, Forsberg M, Björklund MG, Gumbel K, Halimi A, Hallin I, Hamsten C, Hansson M, Hedhammar M, Hercules G, Kampf C, et al:: A human protein atlas for normal and cancer tissues based on antibody proteomics. Mol Cell Proteomics 2005, 4(12):1920-1932.

25. Wellbrock C, Lammers R, Ullrich A, Schartl M: Association between the melanoma-inducing receptor tyrosine kinase Xmrk and src family tyrosine kinases in Xiphophorus. Oncogene 1995, 10(11):2135-2143.

26. Wellbrock C, Schartl M: Activation of phosphatidylinositol 3-kinase by a complex of p59fyn and the receptor tyrosine kinase Xmrk is involved in malignant transformation of pigment cells. Eur J Biochem 2000, 267(12):3513-3522.

27. Maat W, el Filali M, Dirks-Mulder A, Luyten GP, Gruis NA, Desjardins L, Boender P, Jager MJ, van der Velden PA: Episodic Src activation in uveal melanoma revealed by kinase activity profiling. Br J Cancer 2009, 101(2):312-319.

28. Eustace AJ, Crown J, Clynes M, O'Donovan N: Preclinical evaluation of dasatinib, a potent Src kinase inhibitor, in melanoma cell lines. J Trans/ Med 2008, 6:53.

29. Homsi J, Cubitt CL, Zhang S, Munster PN, Yu H, Sullivan DM, Jove R, Messina JL, Daud Al: Src activation in melanoma and Src inhibitors as therapeutic agents in melanoma. Melanoma Res 2009, 19(3):167-175.

30. Boukerche H, Su ZZ, Prevot C, Sarkar D, Fisher PB: mda-9/Syntenin promotes metastasis in human melanoma cells by activating c-Src. Proc Natl Acad Sci USA 2008, 105(41):15914-15919.

31. Packer $L M$, East $P$, Reis-Filho JS, Marais R: Identification of direct transcriptional targets of (V600E)BRAF/MEK signalling in melanoma. Pigment Cell Melanoma Res 2009, 22(6):785-798.

32. Gao K, Zhou H, Zhang L, Lee JW, Zhou Q, Hu S, Wolinsky LE, Farrell J, Eibl G, Wong DT: Systemic disease-induced salivary biomarker profiles in mouse models of melanoma and non-small cell lung cancer. PLoS One 2009, 4(6):e5875.

33. Hoek KS, Schlegel NC, Brafford P, Sucker A, Ugurel S, Kumar R, Weber BL, Nathanson KL, Phillips DJ, Herlyn M, Schadendorf D, Dummer R: Metastatic potential of melanomas defined by specific gene expression profiles with no BRAF signature. Pigment Cell Res 2006, 19(4):290-302

34. Oy GF, Slipicevic A, Davidson B, Solberg Faye R, Maelandsmo GM, Florenes VA: Biological effects induced by insulin-like growth factor binding protein 3 (IGFBP-3) in malignant melanoma. Int $J$ Cancer 126(2):350-361

35. Xi Y, Nakajima G, Hamil T, Fodstad O, Riker A, Ju J: Association of insulinlike growth factor binding protein-3 expression with melanoma progression. Mol Cancer Ther 2006, 5(12):3078-3084.

36. Jeffrey KL, Camps M, Rommel C, Mackay CR: Targeting dual-specificity phosphatases: manipulating MAP kinase signalling and immune responses. Nat Rev Drug Discov 2007, 6(5):391-403.

37. Rangel J, Nosrati M, Torabian S, Shaikh L, Leong SP, Haqq C, Miller JR 3, Sagebiel RW, Kashani-Sabet M: Osteopontin as a molecular prognostic marker for melanoma. Cancer 2008, 112(1):144-150.

38. Haritoglou I, Wolf A, Maier T, Haritoglou C, Hein R, Schaller UC: Osteopontin and 'melanoma inhibitory activity': comparison of two serological tumor markers in metastatic uveal melanoma patients. Ophthalmologica 2009, 223(4):239-243. 
39. Xu L, Shen SS, Hoshida Y, Subramanian A, Ross K, Brunet JP, Wagner SN, Ramaswamy S, Mesirov JP, Hynes RO: Gene expression changes in an animal melanoma model correlate with aggressiveness of human melanoma metastases. Mol Cancer Res 2008, 6(5):760-769.

40. Amit I, Citri A, Shay T, Lu Y, Katz M, Zhang F, Tarcic G, Siwak D, Lahad J, Jacob-Hirsch J, et al:: A module of negative feedback regulators defines growth factor signaling. Nat Genet 2007, 39(4):503-512.

41. Ramnarain DB, Park S, Lee DY, Hatanpaa KJ, Scoggin SO, Otu H, Libermann TA, Raisanen JM, Ashfaq R, Wong ET, Wu J, Elliott R, Habib AA: Differential gene expression analysis reveals generation of an autocrine loop by a mutant epidermal growth factor receptor in glioma cells. Cancer Res 2006, 66(2):867-874.

42. Debinski W, Gibo DM: Fos-related antigen 1 modulates malignant features of glioma cells. Mol Cancer Res 2005, 3(4):237-249.

43. Reisfeld RA, Niethammer AG, Luo Y, Xiang R: DNA vaccines suppress tumor growth and metastases by the induction of anti-angiogenesis. Immunol Rev 2004, 199:181-190.

\section{Pre-publication history}

The pre-publication history for this paper can be accessed here: http://www.biomedcentral.com/1471-2407/10/386/prepub

doi: 10.1186/1471-2407-10-386

Cite this article as: Teutschbein et al., Gene expression analysis after receptor tyrosine kinase activation reveals new potential melanoma proteins BMC Cancer 2010, 10:386

Submit your next manuscript to BioMed Central and take full advantage of:

- Convenient online submission

- Thorough peer review

- No space constraints or color figure charges

- Immediate publication on acceptance

- Inclusion in PubMed, CAS, Scopus and Google Scholar

- Research which is freely available for redistribution

Submit your manuscript at www.biomedcentral.com/submit 